\title{
Padrões de utilização de indicadores de desempenho não- financeiros: Um estudo exploratório nas empresas de Serra Talhada/PE
}

\author{
Antônio André Cunha Callado \\ Doutorado em Administração pela Universidade Federal de Pernambuco - \\ UFPE Professor na Universidade Federal de Pernambuco - UFPE \\ Rua Dom Manoel de Medeiros, s/n. Dois Irmãos. Recife/PE. CEP: 52171- \\ 030 \\ E-mail: andrecallado@yahoo.com.br \\ Aldo Leonardo Cunha Callado \\ Doutorado em Agronegócios pela Universidade Federal do Rio Grande do \\ Sul - UFRGS \\ Professor na Universidade Federal da Paraíba - UFPB \\ Campus I. Cidade Universitária. João Pessoa/PB. CEP: 50740-480 \\ E-mail: aldocallado@yahoo.com.br \\ Luciano Pires de Andrade \\ Mestrado em Administração Rural e Comunicação Rural pela Universidade \\ Federal Rural de Pernambuco - UFRPE \\ Professor na Universidade Federal Rural de Pernambuco - UFRPE \\ Unidade Acadêmica de Serra Talhada. Serra Talhada/PE. CEP: \\ 56900-000 \\ E-mail: luciano@uast.ufrpe.br
}

\section{RESUMO}

Este artigo teve o objetivo de identificar a estrutura e padrões de relações existentes entre os indicadores de desempenho não-financeiro em empresas localizadas em Serra Talhada/PE. Para a realização dessa pesquisa foram analisadas 46 empresas de médio e grande porte que atuam no setor de varejo e investigados 18 indicadores de desempenho. $O$ instrumento utilizado para a coleta de dados foi o questionário. Para atingir o objetivo proposto, foram utilizadas três abordagens metodológicas multivariadas complementares: a análise de agrupamentos, 0 escalonamento multidimensional e a análise fatorial. Os resultados obtidos demonstram que apenas dez indicadores de desempenho puderam ser considerados como pertencentes a algum agrupamento de maneira significativa. Através da análise de agrupamentos foi observada a presença de três agrupamentos, o que foi corroborado pelo escalonamento multi-dimensional. $\mathrm{Na}$ análise fatorial foram encontrados apenas dois fatores. Foram encontradas evidências inerentes aos padrões de relações entre alguns dos indicadores de desempenho não-financeiro investigados.

Palavras-chave: Indicadores de desempenho. Controladoria. Gestão. 
Padrões de utilização de indicadores de desempenho não-financeiros: Um estudo exploratório nas empresas de Serra Talhada/PE Antônio André Cunha Callado, Aldo Leonardo Cunha Callado, Luciano Pires de Andrade

The use of non-financial performance indicators: An exploratory study among companies located in Serra Talhada/PE

\section{ABSTRACT}

The objective of this article was to identify both structure and relations patterns between non-financial performance indicators among companies located at Serra talhada/PE. To develop this research, 46 medium e large retail companies were analyzed and 18 performance indicators were investigated. The instrument used to data collection was a questionnaire. To accomplish the objective proposed, tree multivariate methodologies were used: cluster analysis, multidimensional scaling and factor analysis. The results obtained demonstrated that only ten performance indicators could be considered. Three distinct groups were found through the cluster analysis. The same result was found in the multidimensional scaling, but only two factors were found by the factor analysis. Evidence of relations standards was corroborated by these methods.

Key-words: Performance indicators. Controlling. Management.

\section{INTRODUÇÃO}

A medição de desempenho é uma operação estratégica que pode gerar informações centrais à gestão. Através dela a empresa pode obter relatórios e indicadores que demonstram como ela está em relação às metas estabelecidas, podendo conhecer e controlar seu desempenho econômico-financeiro e sua eficiência operacional, bem como sua capacidade de proporcionar satisfação a todos os stakeholders.

Saber definir o que deve ser medido e avaliado nas diferentes atividades realizadas por uma empresa não é uma tarefa simples. Determinar que medidas devem ser realizadas depende da complexidade do processo que se deseja avaliar, da sua importância em relação às metas estabelecidas pela empresa e da expectativa de posteriores usos gerenciais destas informações.

Neste ambiente, as empresas devem estar aptas a identificar eventuais ameaças e oportunidades que estejam surgindo em seu horizonte gerencial, buscando com isso subsidiar a elaboração de um plano estratégico, que esteja cada vez mais 
Padrões de utilização de indicadores de desempenho não-financeiros: Um estudo exploratório nas

empresas de Serra Talhada/PE

Antônio André Cunha Callado, Aldo Leonardo Cunha Callado, Luciano Pires de Andrade

contextualizado, considerando as especificidades de cada setor econômico, principalmente no que se refere à dinâmica industrial dos setores nos quais atuem.

Gonçalves (2002) ressalta que todas as empresas, de um modo geral, necessitam de um sistema de avaliação de desempenho, uma vez que a realização contínua do processo de avaliação permite que a empresa conheça a eficiência e a eficácia de suas ações, bem como o comportamento das pessoas, os processos e os programas da organização.

De acordo com Rodrigues, Schuch e Pantaleão (2003), os sistemas de indicadores de desempenho são pontos de partida para qualquer ação de melhoria empresarial. Estes autores indicam que o sistema de indicadores de desempenho da empresa deveria ser objeto de planejamento, de modo a cobrir todos os aspectos relevantes para a gestão, garantir sua coerência, bem como sua perfeita adequação aos interesses da organização.

A definição dos indicadores de desempenho a serem utilizados faz parte de uma sequência lógica de procedimentos para desenvolvimento e implementação de um sistema de mensuração e avaliação de desempenho. Estas medidas devem ser orientadas para o futuro, procurando-se definir objetivos que traduzam as metas da organização.

Os indicadores de desempenho são elementos fundamentais para a mensuração de performance, bem como para a definição das variáveis que melhor representam o desempenho geral de uma organização.

Para Miranda e Silva (2002), os indicadores de desempenho desejáveis estão relacionados à definição das mensurações que de fato devem ser consideradas, onde devem ser identificados os atributos de desempenho relevantes que serão adotados como referência de avaliação.

Segundo Bernard (1999), o crescimento da utilização de indicadores nãofinanceiros está relacionado com a incapacidade das medidas financeiras, fornecerem todas as informações necessárias para administrar as empresas, na conjuntura atual do mundo dos negócios. 
Padrões de utilização de indicadores de desempenho não-financeiros: Um estudo exploratório nas

empresas de Serra Talhada/PE

Antônio André Cunha Callado, Aldo Leonardo Cunha Callado, Luciano Pires de Andrade

Aaker (2001) assinala que uma das principais dificuldades na administração estratégica de mercado é o desenvolvimento de indicadores de desempenho que representem convincentemente as projeções de longo prazo.

Tradicionalmente, as abordagens metodológicas contidas nas investigações empíricas deste campo de conhecimento têm considerado predominantemente a análise de relações entre indicadores de desempenho e a performance. Não há referências explícitas na literatura acerca das relações dinâmicas existentes entre indicadores de desempenho. Os padrões de utilização de indicadores de desempenho estão relacionados às práticas gerenciais. Contudo, a ausência de conhecimento sobre as relações existentes entre a utilização de indicadores de desempenho representa uma lacuna importante para este campo investigativo.

A utilização de uma abordagem metodológica multivariada pode prover novas fronteiras investigativas sobre a utilização de indicadores de desempenho, bem como sobre as principais práticas gerenciais estratégicas. Além disso, a literatura apresenta resultados predominantemente referentes à pesquisas conduzidas entre empresas localizadas em grandes centros urbanos.

O objetivo deste artigo é identificar a estrutura e padrões de relações existentes entre os indicadores de desempenho não-financeiro em empresas de médio e grande porte que atuam no setor de varejo localizadas em Serra Talhada/PE.

\section{MEDIÇÃO DE DESEMPENHO ATRAVÉS DE INDICADORES NÃO-FINANCEIROS}

Uma correta definição sobre um indicador de desempenho consiste no primeiro passo para o entendimento de seu significado institucional, bem como a visualização da amplitude de sua aplicação.

MacArthur (1996) conceitua indicador de desempenho como sendo a quantificação de como estão sendo realizadas as atividades com o propósito de compará-las às metas especificadas. Kaplan e Norton (1992) consideram que nenhum indicador de desempenho isolado será capaz de prover uma clara representação da 
Padrões de utilização de indicadores de desempenho não-financeiros: Um estudo exploratório nas

empresas de Serra Talhada/PE

Antônio André Cunha Callado, Aldo Leonardo Cunha Callado, Luciano Pires de Andrade

performance de uma organização, nem poderá focar todas as áreas relevantes ao mesmo tempo.

Faz-se necessário ressaltar a existência de uma perspectiva gerencial atribuída à elaboração, à implantação e ao monitoramento de indicadores de desempenho como suporte referencial estratégico para o processo decisório no âmbito de uma organização.

Sobre o papel dos indicadores de desempenho no processo gerencial das organizações, Martins (2004) afirma que este processo de gestão de desempenho é o meio pelo qual uma organização administra seu desempenho de acordo com as estratégias corporativas e funcionais, bem como seus objetivos.

Neely, Gregory e Platts (1996) definem o indicador de desempenho como sendo um meio utilizado para quantificar a eficiência e/ou a eficácia de uma tomada de decisão feita pela empresa. Um indicador de desempenho, portanto, permite às organizações saberem o que estão fazendo e quais os resultados de suas ações.

Rodrigues, Schuch e Pantaleão (2003) mencionam que os indicadores de desempenho são ferramentas centrais que permitem um acompanhamento das principais variáveis de interesse da empresa e possibilitam o planejamento de ações visando melhorias de desempenho.

Zilber e Fischmann (2002) ressaltam que os indicadores de desempenho permitem verificar a propriedade com que as decisões foram tomadas e, eventualmente, corrigir e readequar o processo vigente de gestão.

Várias são as formas de classificação dos indicadores de desempenho apresentadas pela literatura. Paula e Ichikawa (2002) os classificam em indicadores de qualidade e indicadores de produtividade. Segundo as autoras, os primeiros estão relacionados com a satisfação do cliente, enquanto que os segundos medem 0 desempenho dos diferentes processos de uma organização e estão relacionados com a maneira pela qual são utilizados os recursos disponíveis.

Segundo Macedo e Silva (2004), os métodos de classificação que consideram aspectos financeiros e não financeiros tendem a assumir uma importância especial, visto que o desempenho acaba por ser afetado por variáveis de ambas naturezas. 
Padrões de utilização de indicadores de desempenho não-financeiros: Um estudo exploratório nas

empresas de Serra Talhada/PE

Antônio André Cunha Callado, Aldo Leonardo Cunha Callado, Luciano Pires de Andrade

Os indicadores de desempenho podem ser classificados em termos financeiros e não-financeiros. Para Morissete (1977), indicador financeiro é uma medida quantitativa, expressa em valor monetário, resultante das ações tomadas pelas empresas. Como exemplos de indicadores financeiros têm-se lucro e retorno sobre os investimentos. E o indicador não-financeiro é uma medida quantitativa que não será expressa em valor monetário, ou seja, o indicador não-financeiro é apresentado em uma métrica diferente de uma unidade monetária. Como exemplos de indicadores não-financeiros têm-se participação no mercado e evolução do volume de produção.

Walter, Bornia e Kliemann Neto (2000) afirmam que um dos grandes desafios da gestão empresarial contemporânea é a consideração de valores não-financeiros na avaliação do desempenho.

Kaplan e Norton (1997) argumentam que os indicadores financeiros são inadequados para orientar e avaliar a trajetória da empresa em um ambiente competitivo, pois são indicadores de ocorrência, contam parte, mas não toda a história das ações passadas, bem como não fornecem orientações adequadas para as ações que devem ser realizadas hoje e amanhã para criar valor financeiro futuro.

Banker, Potter e Srinivasan (2000) argumentam que as medidas não-financeiras são melhores indicadores de medidas de desempenho futuro que as medidas financeiras, uma vez que as medidas não-financeiras auxiliam os gestores a focarem suas ações em perspectivas de longo prazo.

Segundo Miranda et al. (2001), a literatura tem mostrado que, no passado, as empresas tomavam decisões baseadas apenas em informações financeiras, obtidas através da sua contabilidade. Entretanto, atualmente, as tomadas de decisões envolvem um maior número de variáveis, exigindo uma grande preocupação entre os gestores com indicadores como: satisfação de clientes, qualidade dos produtos, participação no mercado, retenção de clientes, fidelidade dos clientes, inovação, habilidades estratégicas, entre outros.

Ao sistematizar uma retrospectiva das principais pesquisas realizadas sobre a mensuração de desempenho, Neely, Gregory e Platts (2005) destacam que, por mais claro que seu processo evolucionário possa ser, diversos aspectos ainda estão abertos 
Padrões de utilização de indicadores de desempenho não-financeiros: Um estudo exploratório nas empresas de Serra Talhada/PE Antônio André Cunha Callado, Aldo Leonardo Cunha Callado, Luciano Pires de Andrade

à discussão, pois os resultados apresentam lacunas e incongruências que demandam maior sofisticação dos pressupostos teóricos que dão sustentação a esta área de investigação.

Outro aspecto relevante está associado ao caráter dinâmico do ambiente competitivo que precisa ser considerado quando da definição do elenco de indicadores relevantes de desempenho, bem como dos procedimentos operacionais inerentes ao sistema de mensuração de desempenho.

Embora as medidas financeiras tenham sofrido críticas de estudiosos dedicados a esta temática, elas não devem ser esquecidas quando da avaliação de resultados e desempenhos, pois são provas concretas dos efeitos de todas as outras medidas.

\section{PROCEDIMENTOS METODOLÓGICOS}

Dentro da perspectiva abordada neste trabalho faz-se necessário caracterizar o foco metodológico que será adotado para atingir o objetivo proposto. Esta pesquisa possui uma natureza exploratória. Gil (1999) afirma que as pesquisas exploratórias são importantes, pois elas têm a finalidade de desenvolver, esclarecer e modificar conceitos e ideias, bem como formular problemas mais precisos ou hipóteses pesquisáveis em estudos posteriores.

Nesta mesma vertente, Andrade (1999) considera que uma pesquisa exploratória visa agregar informações sobre um determinado assunto com a finalidade de definir objetivos, formular hipóteses ou descobrir novos enfoques a serem investigados.

Comentando sobre as características das pesquisas exploratórias, Gil (2002) destaca que o planejamento deste tipo de pesquisa é bastante flexível e deve considerar diversas perspectivas sobre o fato a ser estudado.

Sobre as pesquisas exploratórias, Vergara (2003) considera que, dada sua natureza, elas não comportam hipóteses iniciais, mas que podem surgir ao longo da realização da pesquisa. 
Padrões de utilização de indicadores de desempenho não-financeiros: Um estudo exploratório nas

empresas de Serra Talhada/PE

Antônio André Cunha Callado, Aldo Leonardo Cunha Callado, Luciano Pires de Andrade

\subsection{Universo e Amostra Investigada}

De acordo com Leite (1978), com a definição do universo delimita-se o campo de pesquisa em termos temporais, geográficos, setoriais ou qualquer outra dimensão cabível com base na disponibilidade ou obtenibilidade de dados, ou com o fundamento nos objetivos e nos custos da execução da pesquisa.

Para acessar e obter informações sobre o universo a ser investigado, Silver (2000) propõe a utilização de listas especializadas como fonte de consulta. A fonte utilizada na presente pesquisa foi o cadastro do Clube dos Diretores Lojistas (CDL) da cidade de Serra Talhada/PE. O universo desta pesquisa foi composto pelas 167 empresas listadas no cadastro do CDL. Este universo é composto predominantemente por empresas que atuam no setor de varejo.

Para a operacionalização desta pesquisa, foi considerada uma amostra nãoprobabilística intencional. De acordo com Martins (2006), uma amostragem intencional deve ser utilizada quando os elementos que irão compor a amostra são escolhidos intencionalmente.

O critério escolhido para a determinação da amostra foi o tamanho das empresas. Nesse caso foram consideradas apenas as empresas de médio e grande porte, ficando a amostra da pesquisa composta pelas 46 empresas de médio e grande porte do setor varejista de Serra talhada que aceitaram participar da pesquisa e fornecer as informações solicitadas.

Ao comparar a operacionalização de pesquisas a partir da utilização de amostras probabilísticas com amostras não probabilísticas, Stevenson (1986) ressalta que amostras não-probabilísticas proporcionam alternativas úteis, desde que seja elaborado um planejamento cuidadoso para determinar quais elementos da população devem compor a amostra a ser investigada.

Colauto e Beuren (2006) consideram que a determinação de procedimentos de amostragem não-probabilística depende exclusivamente dos critérios considerados pelo pesquisador para a construção das amostras. Nesta mesma perspectiva, Bisquera, Sarriera e Martinez (2004) ressaltam que este método amostral seleciona indivíduos a partir de determinados critérios buscando representatividade da amostra resultante. 
Padrões de utilização de indicadores de desempenho não-financeiros: Um estudo exploratório nas empresas de Serra Talhada/PE Antônio André Cunha Callado, Aldo Leonardo Cunha Callado, Luciano Pires de Andrade

\subsection{Indicadores de Desempenho Investigados}

A presente pesquisa possui aspectos exploratórios que envolvem entrevistas com pessoas que tiveram ou têm experiência prática com o problema pesquisado. $O$ elenco de indicadores de desempenho considerados foi elaborado a partir desta fase exploratória inicial. Para Gil (1999), pesquisas exploratórias são desenvolvidas com o objetivo de proporcionar uma visão geral acerca de determinado fato.

Diante do objetivo proposto nesta pesquisa, foram investigados os seguintes indicadores de desempenho:

- Capacidade de produção;

- Evolução do número de clientes;

- Evolução do número de funcionários;

- Fidelidade dos clientes;

- Inovação;

- Investimento em treinamento;

- Investimento em propaganda;

- Investimento em modernização;

- Investimento em ação social;

- Número de reclamações;

- Participação no mercado;

- Perfil do consumidor;

- Qualidade do produto;

- Rotatividade de empregados;

- Tempo de atendimento ao cliente;

- Tempo para solução de reclamações;

- Valor da marca;

- Volume de vendas. 
Padrões de utilização de indicadores de desempenho não-financeiros: Um estudo exploratório nas

empresas de Serra Talhada/PE

Antônio André Cunha Callado, Aldo Leonardo Cunha Callado, Luciano Pires de Andrade

\subsection{Procedimento da Coleta de Dados}

Para a Coleta de dados desta pesquisa foi utilizada a técnica da entrevista estruturada considerada por Chizzotti (1991), como um tipo de comunicação entre um pesquisador que pretende coletar informações sobre fenômenos e indivíduos que detenham essas informações e possam emiti-las.

Para Gil (2002), a entrevista estruturada se desenvolve a partir de uma relação fixa de perguntas. Esta técnica consiste em fazer uma série de perguntas a um informante, conforme roteiro preestabelecido, onde esse roteiro pode constituir-se de um formulário/questionário que será aplicado da mesma forma a todos os informantes/sujeitos da pesquisa, para que se obtenham respostas para as mesmas perguntas.

O instrumento utilizado para a coleta de dados foi o questionário. Sobre o uso de questionário, Chizzotti (1991) indica que ele é um conjunto de questões pré-elaboradas, sistemática e seqüencialmente dispostas em itens que constituem o tema da pesquisa, com o objetivo de suscitar dos informantes respostas por escrito ou verbalmente sobre 0 assunto que os informantes saibam opinar.

Nesta mesma perspectiva, Gil (2002) afirma que a elaboração de um questionário consiste basicamente em traduzir os objetivos específicos da pesquisa em itens bem redigidos.

Outros aspectos decisivos para a escolha desse instrumento de coleta de dados foram as vantagens apresentadas pela sua utilização que, segundo Richardson (1999), são as seguintes:

a) as respostas a perguntas fechadas são fáceis de codificar;

b) o entrevistado não precisa escrever;

c) as perguntas fechadas facilitam o preenchimento total do questionário.

O questionário utilizado foi desenvolvido a partir da revisão da literatura, bem como de contatos iniciais exploratórios realizados em algumas empresas. Todos os questionários obtidos foram respondidos in loco através de entrevistas estruturadas.

O questionário foi aplicado mediante entrevistas realizadas com os gerentes administradores dos grupos de produtos de raspa de mandioca. O processo de 
Padrões de utilização de indicadores de desempenho não-financeiros: Um estudo exploratório nas empresas de Serra Talhada/PE Antônio André Cunha Callado, Aldo Leonardo Cunha Callado, Luciano Pires de Andrade

aplicação direta diminui a possibilidade de os entrevistados não responderem o questionário ou deixarem de responder alguma pergunta. Essas vantagens são ressaltadas por Richardson (1989), ao acrescentar que no contato direto o pesquisador pode explicar e discutir os objetivos da pesquisa e do questionário, além de responder dúvidas que os entrevistados tenham em certas perguntas.

As entrevistas foram realizadas na sede das empresas investigadas mediante agendamentos prévios. O processo de coleta dos dados foi realizado entre os meses de Fevereiro e Março de 2008.

\subsection{Método de Análise}

Para a identificação da estrutura e padrões de relações existentes entre os diversos indicadores de mensuração de desempenho investigados, nesta pesquisa considerou-se uma abordagem multivariada.

Análise multivariada é definida por Hair et al. (2005) como sendo o conjunto de métodos estatísticos que simultaneamente analisam múltiplas medidas sobre cada um dos indivíduos ou objetos sob investigação.

De acordo com Hair et al. (2005) e Rodrigues e Paulo (2007), o tipo de relação investigada (relação de dependência ou de interdependência) é um fator central para a definição da abordagem multivariada apropriada. A análise de relações de dependência pode ser realizada através das seguintes técnicas:
a) modelagem de equações simultâneas;
b) análise de relações canônicas;
c) análise multivariada de variância;
d) regressão múltipla; e
e) análise discriminante.

Por outro lado, a análise de relações de interdependência pode ser executada através das seguintes técnicas:
a) análise de agrupamentos;
b) análise de correspondência;
c) análise fatorial; e 
Padrões de utilização de indicadores de desempenho não-financeiros: Um estudo exploratório nas

empresas de Serra Talhada/PE Antônio André Cunha Callado, Aldo Leonardo Cunha Callado, Luciano Pires de Andrade

d) escalonamento multidimensional.

Uma vez que o objetivo desta pesquisa consistiu em identificar a estrutura e padrões de relações existentes entre os indicadores de desempenho utilizados por empresas localizadas na cidade de Serra Talhada/PE, a abordagem multivariada que analisa relações de interdependência é a apropriada para assegurar consistência metodológica aos procedimentos executados. Para esta pesquisa foram consideradas três técnicas, a saber:

- Análise de agrupamentos;

- Escalonamento multidimensional; e

- Análise fatorial.

Pohlmann (2007) considera que a análise de agrupamentos (ou conglomerados) se caracteriza por ser descritiva, ateorética e não inferencial, não tendo base estatística para formular inferências sobre uma população com base em uma amostra e sendo usada como técnica exploratória.

O escalonamento multidimensional é definido por Herdeiro (2007) como sendo uma técnica estatística que analisa posicionamentos, comparações de padrão, graus de proximidade e classificações por afinidade entre diversos elementos.

Bezerra (2007) define análise fatorial como sendo uma técnica estatística que busca identificar dimensões de variabilidade comuns existentes entre um conjunto de fenômenos. Sobre os procedimentos referentes à escolha da técnica de rotação de fatores adequada, Hair et al. (2005) afirmam que não há regra específica para a definição da técnica a ser utilizada, mas informam que a técnica mais utilizada é a VARIMAX, por estar disponível na maioria dos aplicativos estatísticos computacionais.

Nesta pesquisa considerou-se a utilização de mais de uma técnica multivariada em função da intenção de caracterizar a estrutura de relações e padrões de uso referentes aos indicadores de mensuração de desempenho no âmbito das empresas investigadas, bem como para comparar os resultados obtidos.

Considerando a possibilidade de utilização de mais de uma técnica, Hair et al. (2005) ressaltam que as técnicas multivariadas que investigam interdependências podem ser comparadas entre si. 
Padrões de utilização de indicadores de desempenho não-financeiros: Um estudo exploratório nas empresas de Serra Talhada/PE Antônio André Cunha Callado, Aldo Leonardo Cunha Callado, Luciano Pires de Andrade

A operacionalização desta pesquisa considerou indicadores de mensuração de desempenho estudados como sendo binários, representando matematicamente o uso (1) e o não uso (zero). Todos os cálculos e demais procedimentos estatísticos foram efetuados a partir do aplicativo computacional STATISTICA for windows.

\subsection{Limitações do Estudo}

Sobre as limitações do estudo, estas podem surgir devido a diferentes fatores, como: metodologia utilizada; características da amostra; base teórica utilizada; tratamento estatístico adotado; etc. Para Creswell (1994), a limitação indica uma fragilidade potencial do estudo. Nesta pesquisa, algumas limitações podem ser destacadas:

- dadas as características apresentadas pela amostra (não-probabilística), os resultados obtidos não permitem generalizações para todas as empresas pertencentes ao setor de varejo da cidade de Serra talhada;

- dadas as características do universo de pesquisa considerado (cadastro disponibilizado pelo $\mathrm{CDL}$ ) há a possibilidade deste não contemplar todas as empresas existentes de fato quando da coleta de dados;

- as técnicas estatísticas utilizadas geram representações estruturais das relações entre os indicadores de mensuração de desempenho investigados, mas não são apropriadas para a realização de inferências estatísticas que vão além dos próprios resultados obtidos.

\section{APRESENTAÇÃO E ANÁLISE DOS RESULTADOS}

Como a análise dos resultados considerou uma perspectiva multivariada, faz-se necessário ressaltar que as duas técnicas utilizadas tiveram o objetivo de ilustrar a estrutura de relações inerentes ao uso dos diversos indicadores de mensuração de desempenho utilizados pelas empresas localizadas na cidade de Serra Talhada.

Este procedimento assume a premissa de que, na medida em que sejam encontradas proximidades entre alguns dos indicadores de mensuração de 
Padrões de utilização de indicadores de desempenho não-financeiros: Um estudo exploratório nas empresas de Serra Talhada/PE Antônio André Cunha Callado, Aldo Leonardo Cunha Callado, Luciano Pires de Andrade

desempenho, estes estejam relacionados entre si. A utilização de mais de uma técnica multivariada (análise de agrupamento e escalonamento multidimensional) para analisar os resultados, foi adotada para dar maior consistência e robustez aos resultados encontrados.

Para a execução da análise de agrupamento, foi primeiramente utilizado o procedimento de árvore de agrupamento. A regra de amalgamação adotada foi referenciada por elos simples e as distâncias foram calculadas a partir do procedimento city-block (manhattan). Os resultados estão dispostos no gráfico 1.

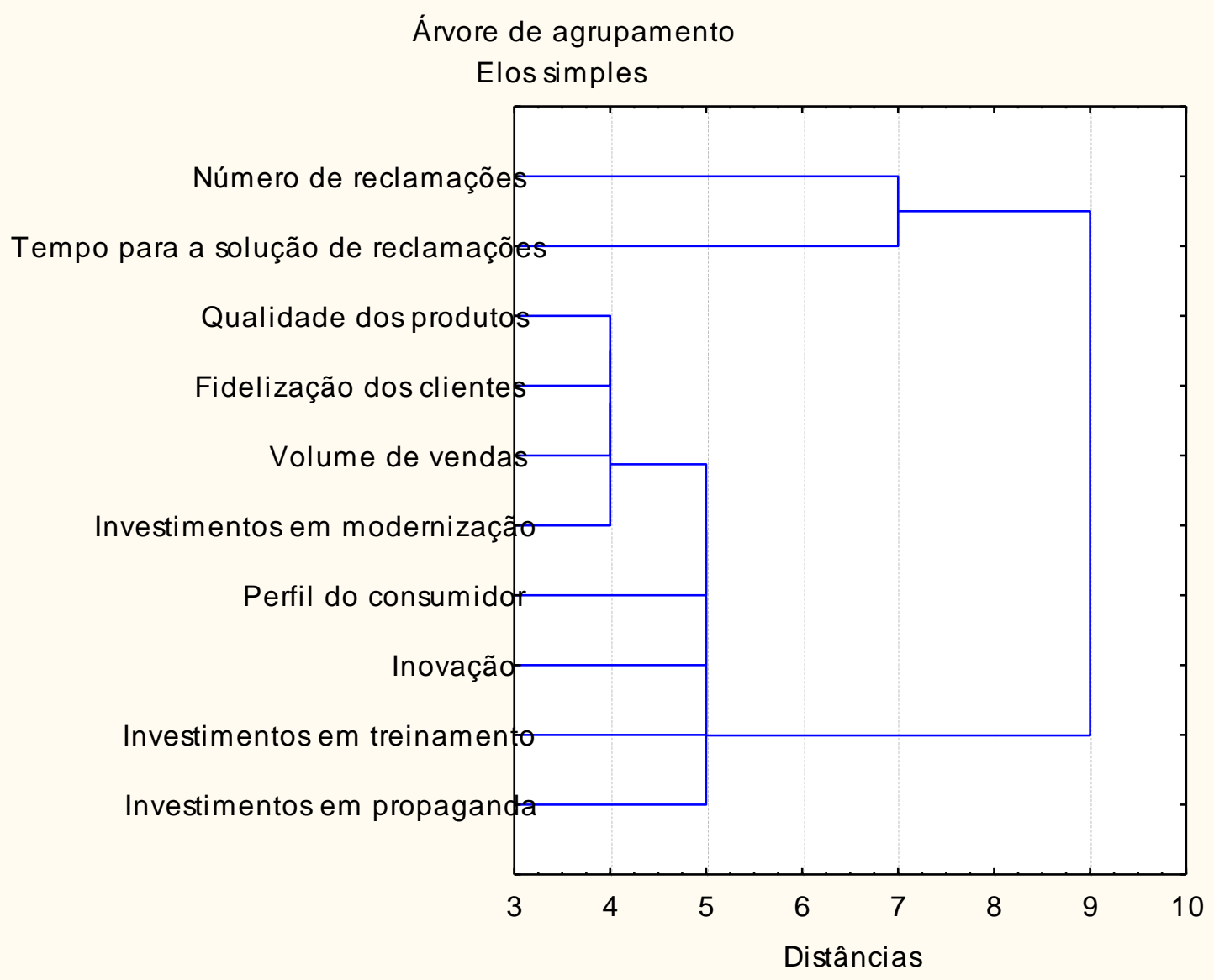

Gráfico 1 - Relação entre as variáveis através da análise de agrupamentos

Fonte: Cálculos próprios.

Número de variáveis: 10

Número de casos: 46

Regra de Amalgamação: Elos simples

Mensuração das Distâncias: Distâncias City-block (Manhattan) 
Padrões de utilização de indicadores de desempenho não-financeiros: Um estudo exploratório nas

empresas de Serra Talhada/PE

Antônio André Cunha Callado, Aldo Leonardo Cunha Callado, Luciano Pires de Andrade

A análise de agrupamentos vai de encontro à perspectiva gerencial atribuída aos indicadores de desempenho proposta por Kaplan e Norton (1992), pois estes autores consideram que nenhum indicador isoladamente seja capaz de prover uma clara representação da performance de uma organização. Desta forma, a análise de agrupamento parece ser uma abordagem metodológica apropriada para suprir esta limitação.

O gráfico gerado mostra os agrupamentos obtidos. Pode-se observar que dos dezoito indicadores de desempenho não-financeiros investigados, apenas dez apresentaram-se relacionados de maneira significativa. Deste modo, os demais indicadores foram excluídos da pesquisa, pois não faria sentido considerá-los na análise dos resultados obtidos através do escalonamento multidimensional. $\mathrm{A}$ árvore de agrupamentos destaca a presença de três grupos significativos de variáveis. Dentre os distintos agrupamentos encontrados, dois agrupamentos apresentam relações internas muito significativas (que se dão no nível 5 da escala de distâncias).

O primeiro é composto pelos indicadores de avaliação de desempenho nãofinanceiro que tratam da qualidade dos produtos, da fidelização dos clientes, do volume de vendas e dos investimentos em modernização. Este agrupamento se caracteriza por um perfil predominantemente facetado pela relação entre as empresas e seus clientes, no qual a qualidade, fidelização e investimentos em modernização estão presentes e, portanto, relacionados ao volume de vendas. A configuração deste agrupamento traduz a relevância e a interdependência das dimensões representadas pela relação entre as empresas investigadas com os seus clientes.

Ao identificar relações significativas entre indicadores de desempenho, conforme é sugerido por Miranda e Silva (2002) como sendo desejáveis e relevantes, os achados obtidos sugerem que os atributos de desempenho mais relevantes a serem adotados como referência de avaliação devem ser identificados de maneira direta e objetiva.

O segundo agrupamento é formado pelas variáveis que representam o perfil do consumidor, as práticas de inovação, os investimentos em treinamento e os investimentos e propaganda. Estas dimensões inerentes à perspectiva dos clientes 
Padrões de utilização de indicadores de desempenho não-financeiros: Um estudo exploratório nas empresas de Serra Talhada/PE Antônio André Cunha Callado, Aldo Leonardo Cunha Callado, Luciano Pires de Andrade

caracterizam outro foco gerencial central das operações desenvolvidas pelas empresas investigadas, uma vez que fica evidente a estratificação de seus clientes. Este agrupamento de indicadores de desempenho ilustra a relevância da segmentação de mercado para monitorar a percepção e os hábitos de consumo dos clientes.

O terceiro agrupamento encontrado é composto pelos indicadores que dimensionam o número de reclamações e o tempo para a solução de reclamações. A intensidade das relações encontradas no núcleo deste agrupamento não foi tão significativa quanto às demais (se deu no nível 7 da escala de distâncias), mas revela a importância do suporte pósvenda.

Dando continuidade aos procedimentos de análise propostos para esta pesquisa, foi investigada a disposição multidimensional das relações entre os dez indicadores de desempenho não-financeiros considerados como válidos pela análise de agrupamentos para corroborar ou refutar os achados obtidos através da análise de agrupamentos.

Em seguida foi elaborado um gráfico bi-dimensional. Desta forma, a representação espacial da disposição das variáveis foi importante para identificar a disposição espacial das variáveis e verificar se há similaridade com a composição dos agrupamentos obtidos. Os resultados estão dispostos no gráfico 2 . 
Padrões de utilização de indicadores de desempenho não-financeiros: Um estudo exploratório nas empresas de Serra Talhada/PE Antônio André Cunha Callado, Aldo Leonardo Cunha Callado, Luciano Pires de Andrade

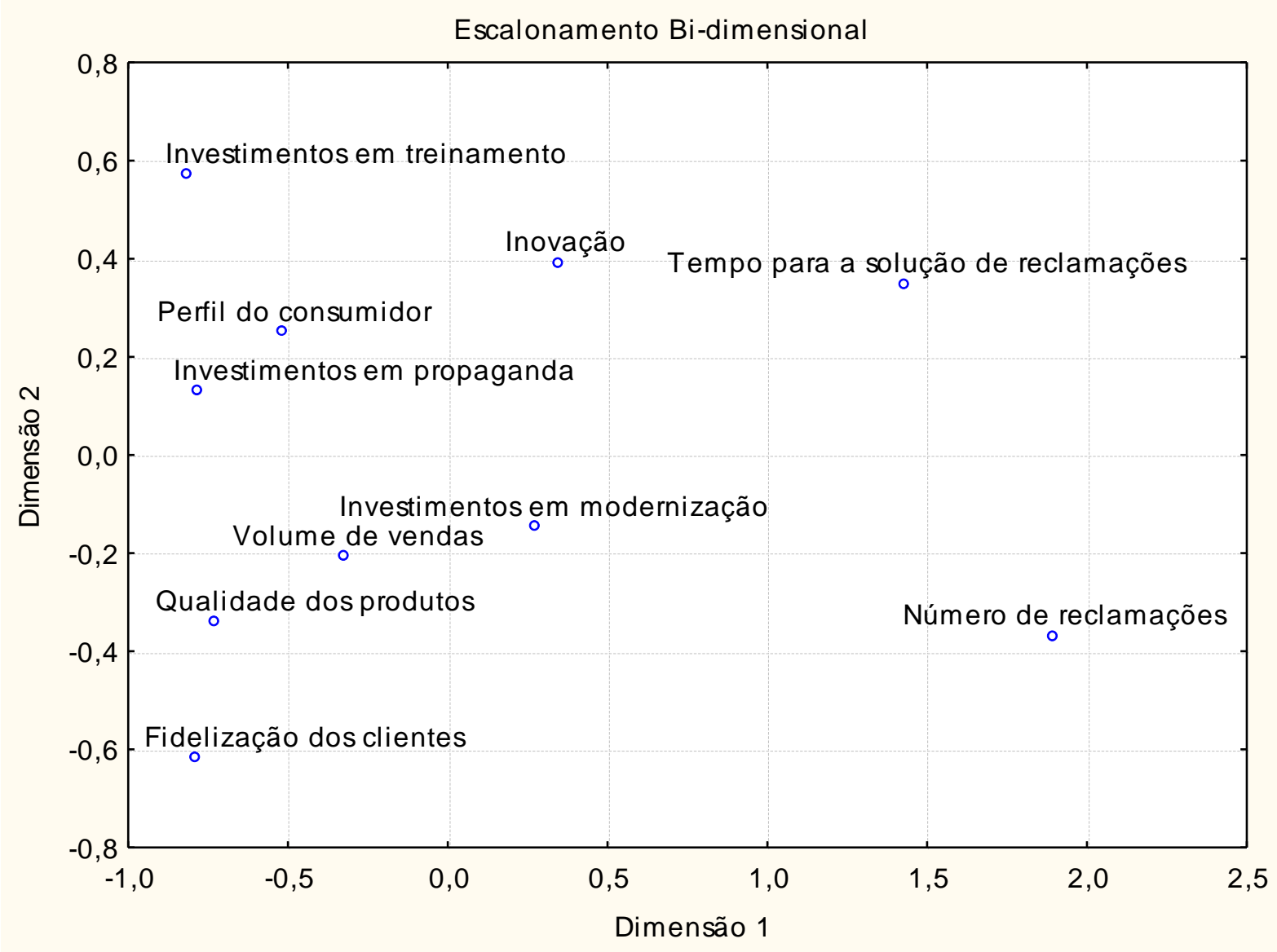

Gráfico 2 - Agrupamento das variáveis através do escalonamento bi-dimensional

Fonte: Cálculos próprios.

Número de variáveis: 10

Número de dimensões: 2

Última interação computada: 82; Melhor iteração: 46

Alienação: 0,1291284

Estresse: 0,0858381

Os resultados apresentados através do gráfico bi-dimensional obtido corroboram a proximidade de relações entre os indicadores de mensuração de desempenho dentro de cada um dos agrupamentos encontrados anteriormente, bem como confirma 0 relativo distanciamento entre eles.

Cada um dos indicadores de mensuração de desempenho pode ser visualizado individualmente através da distribuição espacial gerada pelo escalonamento bidimensional. Estes resultados, embora coerentes com os resultados anteriores, não 
Padrões de utilização de indicadores de desempenho não-financeiros: Um estudo exploratório nas empresas de Serra Talhada/PE Antônio André Cunha Callado, Aldo Leonardo Cunha Callado, Luciano Pires de Andrade

podem ser considerados como estatisticamente significativos, uma vez que os níveis de alienação e estresse ficaram próximos a 0,10.

Com a finalidade de corroborar os resultados obtidos e dar maior precisão à representação gráfica do escalonamento multidimensional, os cálculos foram executados novamente para gerar um gráfico tri-dimensional.

\section{Escalonamento Tri-dimensional}

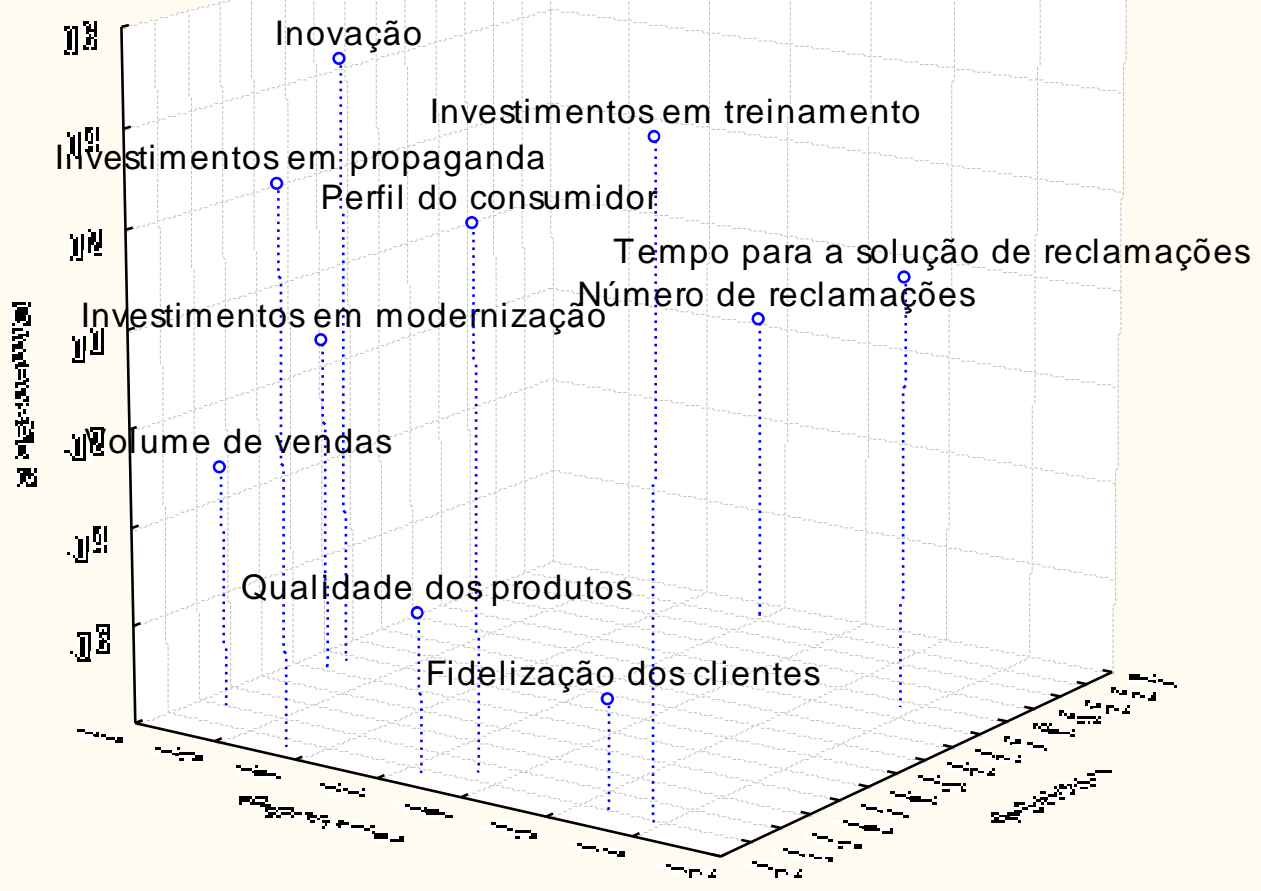

Gráfico 3 - Agrupamento das variáveis através do escalonamento tri-dimensional Fonte: Cálculos próprios.

Número de variáveis:10

Número de dimensões: 3

Última interação computada: 252; Melhor iteração: 81.

Alienação: 0,0773587

Estresse: 0,0449253

O escalonamento tri-dimensional apresenta com maior nitidez a proximidade do relacionamento entre os diversos indicadores não-financeiros considerados. Dentro do 
Padrões de utilização de indicadores de desempenho não-financeiros: Um estudo exploratório nas empresas de Serra Talhada/PE Antônio André Cunha Callado, Aldo Leonardo Cunha Callado, Luciano Pires de Andrade

primeiro agrupamento, a fidelização dos clientes se mostrou bastante próximo à qualidade dos produtos, enquanto o volume de vendas se mostrou mais próximo aos investimentos em modernização. Este resultado demonstra que existem particularidades distintas no âmbito deste agrupamento.

Em relação ao segundo agrupamento, percebe-se uma maior proximidade entre o perfil do consumidor e os investimentos em propaganda. Enquanto os investimentos em treinamento e a inovação não se posicionaram tão próximos entre si.

Finalmente, o número de reclamações e o tempo para a solução de reclamações (indicadores não financeiros que compõem o terceiro agrupamento) se posicionaram de maneira relativamente próxima, contudo sem poder configurar um relacionamento estreito entre eles.

E por último foi executada a análise fatorial. A configuração do cálculo ainda considerou a busca por três fatores, uma vez que as duas técnicas multivariadas anteriores evidenciaram a presença de três agrupamentos distintos, exigindo um mínimo eigenvalue equivalente a 1 . Os resultados da análise fatorial não rotacionada estão apresentados na tabela 1. 
Padrões de utilização de indicadores de desempenho não-financeiros: Um estudo exploratório nas empresas de Serra Talhada/PE Antônio André Cunha Callado, Aldo Leonardo Cunha Callado, Luciano Pires de Andrade

Tabela 1 - Resultados da análise fatorial não-rotacionada

\begin{tabular}{||c|c|c|}
\hline \multicolumn{1}{|c|}{ Indicadores não-financeiros } & Fator 1 & Fator 2 \\
\hline \hline Número de reclamações & $-0,021990$ & 0,908616 \\
\hline \hline Tempo para a solução de reclamações & $-0,117722$ & 0,883886 \\
\hline \hline Qualidade dos produtos & $-0,901766$ & $-0,281172$ \\
\hline \hline Fidelização dos clientes & $-0,830685$ & $-0,179508$ \\
\hline \hline Perfil do consumidor & $-0,937048$ & $-0,227465$ \\
\hline \hline Volume de vendas & $-0,900069$ & $-0,023781$ \\
\hline \hline Inovação & $-0,632142$ & 0,581805 \\
\hline \hline Investimentos em treinamento & $-0,829228$ & $-0,097808$ \\
\hline Investimentos em propaganda & $-0,925640$ & $-0,122673$ \\
\hline Investimentos em modernização & $-0,669503$ & $\mathbf{0 , 5 0 7 9 2 0}$ \\
\hline Capacidade explicativa & $\mathbf{0 , 5 5 9 8 0 1}$ & $\mathbf{0 , 2 3 9 1 5 2}$ \\
\hline
\end{tabular}

Fonte: Cálculos próprios.

Método: Não-rotacionado

Número de variáveis: 10

Método: Componentes principais

Log (10) Determinante da matriz de correlação: $-6,9027$

Número de fatores extraídos: 2

Eigenvalues: Fator 1: 5,59801

Fator 2: 2,39152

Pode-se observar que foram identificados apenas dois fatores. O primeiro fator é composto por indicadores de mensuração de desempenho pertencentes aos dois agrupamentos que obtiveram os mais elevados graus de relacionamento (excetuando inovação e investimento em modernização). O segundo fator é composto pelos indicadores de mensuração de desempenho que representam o número de reclamações e o tempo para a solução das reclamações.

Os resultados obtidos através da análise fatorial demonstram que os três agrupamentos de indicadores de mensuração de desempenho encontrados podem ser considerados como pertencentes a duas categorias distintas.

Para dar mais consistência à análise fatorial realizada, o procedimento foi repetido considerando a rotação dos fatores. O método rotacional utilizado foi o Varimax. Os resultados estão dispostos na tabela 2. 
Padrões de utilização de indicadores de desempenho não-financeiros: Um estudo exploratório nas empresas de Serra Talhada/PE Antônio André Cunha Callado, Aldo Leonardo Cunha Callado, Luciano Pires de Andrade

Tabela 2 - Resultados da análise fatorial rotacionada

\begin{tabular}{||c||c|c|}
\hline \multicolumn{1}{|c||}{ Indicadores não-financeiros } & Fator 1 & Fator 2 \\
\hline \hline Número de reclamações & $-0,177045$ & 0,891472 \\
\hline \hline Tempo para a solução de reclamações & $-0,078223$ & 0,888254 \\
\hline \hline Qualidade dos produtos & 0,941410 & $-0,077372$ \\
\hline \hline Fidelização dos clientes & 0,849836 & 0,006307 \\
\hline \hline Perfil do consumidor & 0,964107 & $-0,017255$ \\
\hline Volume de vendas & 0,883522 & 0,173430 \\
\hline \hline Inovação & 0,489766 & 0,705855 \\
\hline \hline Investimentos em treinamento & 0,830565 & 0,085715 \\
\hline \hline Investimentos em propaganda & 0,930080 & 0,082514 \\
\hline Investimentos em modernização & 0,542366 & 0,641916 \\
\hline Capacidade explicativa & $\mathbf{0 , 5 4 4 4 9 7}$ & $\mathbf{0 , 2 5 4 4 5 6}$ \\
\hline
\end{tabular}

Fonte: Cálculos próprios.

Método: Rotacionado

Número de variáveis: 10

Método: Componentes principais

Log (10) Determinante da matriz de correlação: -6,9027

Número de fatores extraídos: 2

Eigenvalues: Fator 1: 5,59801

Fator 2: 2,39152

Pode-se observar que, novamente, foram identificados apenas dois fatores distintos. O primeiro fator é composto pelos mesmos indicadores de mensuração de desempenho obtidos na primeira análise fatorial não-rotacionada realizada. Por outro lado, a composição do segundo fator obtido através da rotação foi distinta da anterior. Desta vez, além do número de reclamações e do tempo para a solução das reclamações, o indicador referente à inovação também se mostrou presente neste fator.

Estes resultados fornecem evidências empíricas significativas para a compreensão da relação dinâmica entre os indicadores de mensuração de desempenho não-financeiro investigados, bem como demonstram que a identificação da estrutura de relações inerentes à sua utilização no âmbito das empresas localizadas na cidade de Serra Talhada pode gerar informações relevantes para auxiliar o entendimento de suas práticas gerenciais. 
Padrões de utilização de indicadores de desempenho não-financeiros: Um estudo exploratório nas

empresas de Serra Talhada/PE

Antônio André Cunha Callado, Aldo Leonardo Cunha Callado, Luciano Pires de Andrade

Embora os resultados obtidos não possam ser extrapolados para todas as empresas do universo investigado, os achados observados nesta pesquisa exploratória sugerem que este campo de investigação ainda possui algumas vertentes que podem ser exploradas com maior profundidade.

\section{CONCLUSÃO}

Este artigo teve o objetivo de identificar a estrutura e padrões de relações existentes entre os indicadores de desempenho não-financeiro entre as empresas localizadas em Serra Talhada/PE. Para atingir o objetivo proposto foi utilizada uma abordagem metodológica multivariada composta pela utilização de duas técnicas distintas e complementares entre si (análise de agrupamento e escalonamento multidimensional).

Estas técnicas foram consideradas dentro de uma perspectiva comparativa na qual os resultados iguais obtidos teriam maior robustez ao ilustrar as relações entre dezoito indicadores de mensuração de desempenho analisados. Os resultados obtidos demonstraram que apenas sete indicadores de desempenho puderam ser considerados como pertencentes a algum agrupamento de maneira significativa.

Através da árvore de agrupamentos foi observada a presença de três agrupamentos significativos de indicadores de mensuração de desempenho. Resultados semelhantes foram obtidos pelo escalonamento multidimensional.

O escalonamento multidimensional apresentou resultados semelhantes aos encontrados na análise de agrupamentos, destacando a composição desses três agrupamentos. A partir desta perspectiva metodológica, foram encontradas evidências que sugerem que o primeiro grupo possui elos de ligação mais significativos entre os indicadores de avaliação de desempenho não-financeiros que o compõem, em comparação aos demais grupos encontrados.

Ao longo da apresentação dos resultados, observou-se a presença de distintas dimensões gerenciais representadas pelos grupos identificados e que fazem parte do foco estratégico da gestão, bem como revelam indícios particulares referentes à 
Padrões de utilização de indicadores de desempenho não-financeiros: Um estudo exploratório nas

empresas de Serra Talhada/PE

Antônio André Cunha Callado, Aldo Leonardo Cunha Callado, Luciano Pires de Andrade

caracterização da dinâmica das relações entre os principais indicadores de desempenho não-financeiros utilizados pelas empresas investigadas.

Conforme apresentado na literatura, a análise do desempenho pressupõe uma perspectiva múltipla e complementar de diversas perspectivas. Os resultados obtidos corroboram este postulado teórico e dão sustentação à necessidade gerencial de construir um elenco de indicadores de desempenho que seja diversificado, mas que considere os aspectos mais relevantes para o setor no qual a empresa atua.

Foram identificados dois fatores distintos na análise fatorial. O primeiro fator é composto por variáveis encontradas nos dois primeiros agrupamentos, enquanto o segundo fator foi formado pelas variáveis que dão sustentação ao núcleo do terceiro agrupamento.

Apesar dos resultados obtidos serem estatisticamente significativos, não há sustentação suficiente para se afirmar categoricamente que os três grupos encontrados sejam independentes entre si ou que eles não exerçam contribuições individuais complementares para com o monitoramento do desempenho das empresas investigadas.

Embora os resultados obtidos nesta pesquisa não possam ser generalizados para todas as empresas pertencentes ao universo investigado, eles apontam evidências relevantes sobre os padrões de relacionamento entre alguns dos diversos indicadores de desempenho utilizados pelas empresas investigadas.

Estudos mais aprofundados poderão gerar informações mais detalhadas sobre a natureza das relações dinâmicas encontradas nesta pesquisa, e contribuir para o aprimoramento da base de conhecimento existente sobre esta importante atividade econômica.

\section{REFERÊNCIAS}

AAKER, D. A. (2001). Administração Estratégica de Mercado. (5 ed.). Porto Alegre, Bookman. 352 p.

ANDRADE, M. M. (1999). Introdução à metodologia do trabalho científico. (4 ed.). São Paulo, Atlas. 174 p. 
Padrões de utilização de indicadores de desempenho não-financeiros: Um estudo exploratório nas

empresas de Serra Talhada/PE

Antônio André Cunha Callado, Aldo Leonardo Cunha Callado, Luciano Pires de Andrade

BANKER, R. D; POTTER, G. e SRINIVASAN, D. (2000). An empirical investigation of an incentive plan that includes nonfinancial performance measures. The Accounting Review, Florida, 75(1):65-92.

BERNARD, R. R. S. (1999). The rise of non-financial measures. In: Encontro da Associação Nacional dos Programas de Pós-Graduação em Administração, 23, Foz do Iguaçu. Anais... Foz do Iguaçu: ANPAD. 15 p.

BEZERRA, F. A. (2007). Análise fatorial. São Paulo, Atlas. In: Corrar, Luiz J; Paulo, Edílson; Dias Filho, José Maria (Org.). Análise Multivariada. 344 p.

CHIZZOTTI, A. A. (1991). Pesquisa em Ciências Humanas e Sociais. São Paulo, Cortez. $164 \mathrm{p}$.

COLAUTO, R. D. e BEUREN, I. M. (2006). Coleta, análise e interpretação dos dados. (3 ed.). São Paulo, Atlas. In: Beuren, I. M. (Org.). Como elaborar trabalhos monográficos em contabilidade. $200 \mathrm{p}$.

CRESWELL, J. W. (1994). Research Design: qualitative \& quantitative approaches. Thousand Oaks, Sage Publications. 228 p.

GIL, A. C. (1999). Métodos e Técnicas de Pesquisa Social. (5 ed.). São Paulo: Atlas.

$159 \mathrm{p}$. . (2002). Como elaborar projetos de pesquisa. (4 ed.). São Paulo, Atlas,

GONÇALVES, J. P. (2002). Desempenho Organizacional. Seminário Econômico. São Paulo, no 815. 2 p.

HAIR, J. F.; ANDERSON, R. E.; TATHAM R. L.; BLACK, W. C. (2005). Análise Multivariada de Dados. Porto Alegre: Bookman, 2005, 593 p.

HERDEIRO, R. F. C. (2007). Escalonamento multidimensional. São Paulo, Atlas. In: Corrar, Luiz J; Paulo, Edílson; Dias Filho, José Maria (Org.). Análise Multivariada. 344 P.

KAPLAN, R. S e NORTON, D. P. (1992). The Balanced Scorecard - The measures that drive performance. The Harvard Business Review, January-February. $12 \mathrm{p}$.

KAPLAN, R. S e NORTON, D. P. A. (1997). Estratégia em ação: Balanced Scorecard. (9 ed.). Rio de Janeiro, Campus. 344 p. 
Padrões de utilização de indicadores de desempenho não-financeiros: Um estudo exploratório nas

empresas de Serra Talhada/PE

Antônio André Cunha Callado, Aldo Leonardo Cunha Callado, Luciano Pires de Andrade

LEITE, J. A. A. (1978). Metodologia de Elaboração de Teses. São Paulo, McGraw-Hill do Brasil. $122 \mathrm{p}$.

MACARTHUR, J. B. (1996). Performance measures that count: monitoring variables of strategic importance. Journal of Cost Management, 10(3):39-45.

MACEDO, M. A. S e SILVA, F. F. (2004). Análise de desempenho organizacional: utilizando indicadores financeiros e não financeiros na avaliação de performance empresarial. In: Encontro da Associação Nacional dos Programas de Pós-Graduação em Administração, 28. Curitiba. Anais... Curitiba, ANPAD.

MARTINS, D. M. (2004). Eficácia dos indicadores de desempenho na cadeia de fornecimento automotivo. In: Simpósio de Administração da Produção, Logística e Operações Internacionais, 7, São Paulo. Anais... São Paulo, SIMPOI.

MARTINS, G. A. (2006). Estatística geral e aplicada. (3 ed.). São Paulo, Atlas. 428 p.

MIRANDA. L. C.; SILVA, J. D.; CAVALCANTI, R. F. V.; AQUINO, E. M.; FELOWS C. P. (2001). Olhando para fora da empresa: combinando "Balanced Scorecard" com "Supply Chain Management" para considerar o fornecedor na medição de desempenho. In: Encontro da Associação Nacional dos Programas de Pós-Graduação em Administração, 25, Campinas. Anais... Campinas, ANPAD.

MIRANDA. L. C. e SILVA, J. D. G. (2002). Medição de Desempenho. In: SCHMIDT, Paulo (Org.). Controladoria: Agregando Valor para a Empresa. Porto Alegre, Bookman. $262 \mathrm{p}$.

MORISSETE, R. (1997). Toward a theory of information choices in organizations: an integrative approach. Ph.D. Dissertation in Accounting. University of Waterloo. 329 p.

NEELY, A; GREGORY, M; PLATTS, K. (2005). The evolution of performance measurement research: Developments in the last decade and a research agenda for the next. International Journal of Operations \& Production Management, vol. 25, n. 12.

System Design: should Process Based Approaches be adopted? International Journal Production Economics, Amsterdam, 46(47). 9 p.

PAULA, R. N. C. e ICHIKAWA, E. Y. (2002). Indicadores de produtividade em cooperativas do Paraná: um estudo comparativo de casos. In: Encontro da Associação Nacional dos Programas de Pós-Graduação em Administração, 26. Salvador. Anais... Salvador, ANPAD. 
Padrões de utilização de indicadores de desempenho não-financeiros: Um estudo exploratório nas empresas de Serra Talhada/PE Antônio André Cunha Callado, Aldo Leonardo Cunha Callado, Luciano Pires de Andrade

POHLMANN, M. C. (2007). Análise de Conglomerados. In: Corrar, Luiz J; Paulo, Edílson; Dias Filho, José Maria (Org.). Análise Multivariada. São Paulo, Atlas. 344 p.

RICHARDSON, R.J. (1999). Pesquisa social: métodos e técnicas. São Paulo, Atlas. 334 p.

RODRIGUES, L. H.; SCHUCH, C. e PANTALEÃO, L. H. (2003). Uma abordagem para construção de sistemas de indicadores alinhando a teoria das restrições e o Balanced Scorecard. In: Encontro da Associação Nacional dos Programas de Pós-Graduação em Administração, 27. Atibaia. Anais... Atibaia, ANPAD.

RODRIGUES, A. e PAULO, E. (2007). Introdução à análise multivariada. In: Corrar, Luiz J; Paulo, Edílson; Dias Filho, José Maria (Org.). Análise Multivariada. São Paulo, Atlas. $344 \mathrm{p}$.

SILVER, M. (2000). Estatística para Administração. São Paulo, Atlas. 464 p.

VERGARA, S. C. (2003). Projetos e relatórios de pesquisa em administração. (4 ed.). São Paulo, Atlas. 96 p.

WALTER, F.; BORNIA, A. C. e KLIEMANN NETO, F. J. (2000). Análise comparativa de duas metodologias para elaboração do Balanced Scorecard. In: Encontro da Associação Nacional dos Programas de Pós-Graduação em Administração, 24, Florianópolis. Anais... Florianópolis, ANPAD.

ZILBER, M. A. e FISCHMANN, A. A. (2002). Competitividade e a importância de indicadores de desempenho: utilização de um modelo de tendência. In: Encontro da Associação Nacional dos Programas de Pós-Graduação em Administração, 26. Salvador. Anais... Salvador, ANPAD.

Data de Submissão: 13/05/2008

Data de Aceite: 17/07/2008 